\title{
A Fast and Intelligent Automatic Power Control for a GPON Burst-Mode Optical Transmitter
}

\author{
Dieter Verhulst, Johan Bauwelinck, Yves Martens, Xing-Zhi Qiu, and Jan Vandewege
}

\begin{abstract}
A generic 1.25-Gb/s burst-mode optical transmitter with an intelligent automatic power control has been developed and tested successfully. The chip quickly adjusts the optical power during initialization from safe preset values to the required high and low levels with a guaranteed extinction ratio, and accurately tracks level drifts during data transmission. The measured performance complies with the ITU-T gigabit passive optical network standards including the power leveling mechanism option.
\end{abstract}

Index Terms-Automatic power control (APC), burst-mode laser driver, burst-mode optical transmitter, gigabit passive optical network (GPON).

\section{INTRODUCTION}

$\mathbf{T}$ HE burst-mode optical transmitter (BM-TX) located in each subscriber optical network unit (ONU) is one of the key building blocks of a gigabit passive optical network (GPON) [1]. Individual laser characteristics show a wide spread, a strong temperature dependence, and a nonlinear relationship between laser current and optical power. So both the laser modulation (IMOD) and bias current (IBIAS) must be regulated to reach the target (average) optical power and the required extinction ratio (ER). A dual-loop automatic power control (APC) is required to stabilize the optical output power levels, over temperature and age, within the GPON physical media-dependent layer specifications defined in ITU-T Recommendation G.984.2 [2].

A PON operates in a point-to-multipoint time-division multiple access scheme with maximum 32 talkers. The BM-TX must not send upstream light in timing windows allocated to other ONUs. Therefore, the BM-TX power level must be stabilized quickly within the short time slots allocated to the specific ONU. Previous burst-mode APC approaches are either laborious, requiring multiple temperature-dependent calibration values to be stored in look-up tables, or too slow for optical power regulation in a GPON [3]. In [4], a current-mode architecture was presented that overcomes these drawbacks. However, the APC algorithm was implemented in an FPGA <<AUTHOR: PLEASE SPELL OUT FPGA>>, compromising the regulation speed. In order to support fast regulation of an ONU during an initialization phase and a fast network recovery after a network failure, an innovative $1.25-\mathrm{Gb} / \mathrm{s} \mathrm{BM-TX}$ with an on-chip digital APC loop (and improved circuitry) was developed.

The power leveling mechanism (PLM) we implemented in this BM-TX extends the BM-TX laser lifetime, and relaxes the dynamic range requirement for the burst-mode receiver

Manuscript received April 20, 2005; revised July 15, 2005.

The authors are with Ghent University, INTEC/IMEC, Gent B-9000, Belgium (e-mail: dieter.verhulst@intec.ugent.be; johan.bauwelinck@intec.ugnet.be; xingzhi@intec.ugent.be; jan.vandewege@intec.ugent.be).

Digital Object Identifier 10.1109/LPT.2005.857577
TABLE I

MEAN LAUNCHED POWER SPECIFICATION FOR 1.25-Gb/s Class B GPON BM-TX WITH PLM OPTION

\begin{tabular}{lccc}
\hline \multicolumn{1}{c}{ PLM Mode } & Min & Max & Unit \\
\hline Mode 0 (default) & -2 & +3 & $\mathrm{dBm}$ \\
Mode 1 & -5 & 0 & $\mathrm{dBm}$ \\
Mode 2 & -8 & -3 & $\mathrm{dBm}$ \\
\hline
\end{tabular}

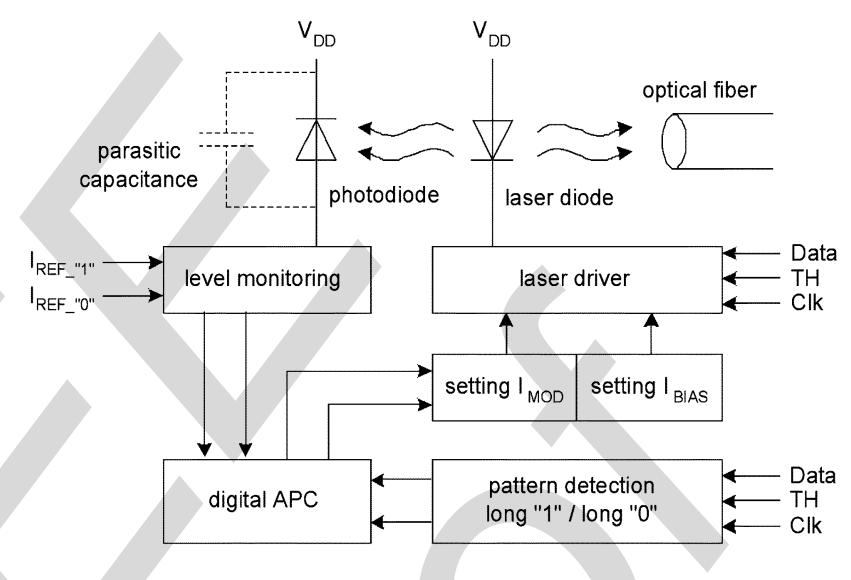

Fig. 1. Simplified block diagram of the GPON ONU BM-TX.

located in the optical line termination (OLT) by reducing the 21-dB burst-to-burst amplitude difference. The PLM adopted in ITU-T Recommendation G.984.2 allows an OLT to request a specific ONU to change its output power in two steps of $3 \mathrm{~dB}$, when the incoming signal level is too strong or too weak. In [5], a power control technique over an EPON $<<$ AUTHOR: PLEASE SPELL OUT EPON $>$ network was proposed in which IMOD is controlled over the network by a single feedback loop. In a GPON supporting the PLM option there are two feedback loops, as each ONU has its own local APC loop to set the optical output to the level requested by the outer PLM loop. Table I lists the BM-TX average output power for the different PLM modes. Mode 0 is the default mode or the mode for a BM-TX that does not support the PLM. The APC loop in a BM-TX with the PLM option must be able to dynamically set the optical output power to the three predefined PLM levels. Moreover, in all three modes, a minimum ER of $10 \mathrm{~dB}$ has to be guaranteed. This letter describes the implementation and test results of a fast and accurate APC approach and its algorithm.

\section{FAST AND ACCURATE APC APPROACH}

Fig. 1 depicts the building blocks of the BM-TX. The laser driver stage is digitally programmable with a 10-bit IBIAS and IMOD setting, respectively. The launched optical power is monitored by capturing the laser back facet light with a photodiode (PD), generating an output current proportional to the optical 
output power with low temperature and age dependency. The parasitic capacitance of such a PD can be up to $15 \mathrm{pF}$ and forms the main speed-limiting factor for any monitoring circuit. In continuous-wave laser drivers, the optical power is regulated by means of slow averaging circuits, but this requires an equal distribution of " 0 " and "1" bits over time, which is not guaranteed in the GPON upstream path.

Due to the PD capacitance, a number of consecutive identical bits is required to detect small variations of the optical level. So the circuitry in [6] required five consecutive bytes for each level measurement. The improved circuitry presented here requires only two bytes for the " 1 " level and four bytes for the " 0 " level.

A pattern detection block continuously scans the data stream for series of consecutive 0 's or 1's, during which accurate measurements can be made by the level monitoring block. The level monitoring block compares the photocurrent with two reference currents (IREF_"0" and IREF_"1") corresponding to the desired "0" and "1" optical level. The pattern detection block samples the complementary metal-oxide-semiconductor (CMOS) outputs of the level monitoring block (indicating that the emitted level is too high or too low) at the correct moments and passes these values to the digital APC algorithm. Subsequently, the digital APC algorithm calculates new settings for the IBIAS and the IMOD current. Due to the pattern detection block, no time-critical signal such as a preamble envelope or an arming signal is required by the APC loop, as all time critical signals are generated on-chip based on the data pattern [7].

In the ITU-T Recommendation G.984.3 "Transmission Convergence Layer for Gigabit Passive Optical Networks," two overheads are available for the ONU to transmit the required patterns of consecutive identical bits. At ONU initialization or when a new PLM level is requested, the OLT allows the ONU to transmit a power leveling sequence upstream (PLSu) field. The content of the 120-byte PLSu overhead can be optimized by every vendor so the transmission of several consecutive " 0 " and " 1 "-bit patterns is guaranteed. The second overhead occurs more frequently and utilizes idle physical layer OAM upstream (PLOAMu) fields. Each ONU is asked regularly (millisecond scale) to send a ten-byte PLOAMu message. If the ONU has nothing to report, it can send a message with five bytes of consecutive 1's and five bytes of consecutive 0 's, so the OLT can track the optical power level during normal operation.

The reference currents (IREF_" 0 " and IREF_"1") are determined by a calibration at room temperature. The " 0 " level was calibrated to $-13.5 \mathrm{dBm}$ to properly bias the $\mathrm{LD}$ and to guarantee an ER above $10 \mathrm{~dB}$ in all PLM modes. The "1" level was calibrated to $-2.5 \mathrm{dBm}$ (PLM Mode 2). Depending on the PLM setting, IREF_"1" is multiplied by two or four. The reference currents are set by two 8-bit current DACs $<<$ AUTHOR: PLEASE SPELL OUT DAC $>>$ to allow a simple automatic calibration. The calibrated values for the different PLM modes are stored in on-chip registers.

\section{Digital APC Algorithm}

During ONU initialization, the APC is in its fast mode to settle quickly to the required optical output power. In this mode, the APC applies an adapted binary search algorithm. Fig. 2 shows how starting from a safe preset current, the current is gradually increased until it is found too high and a binary search

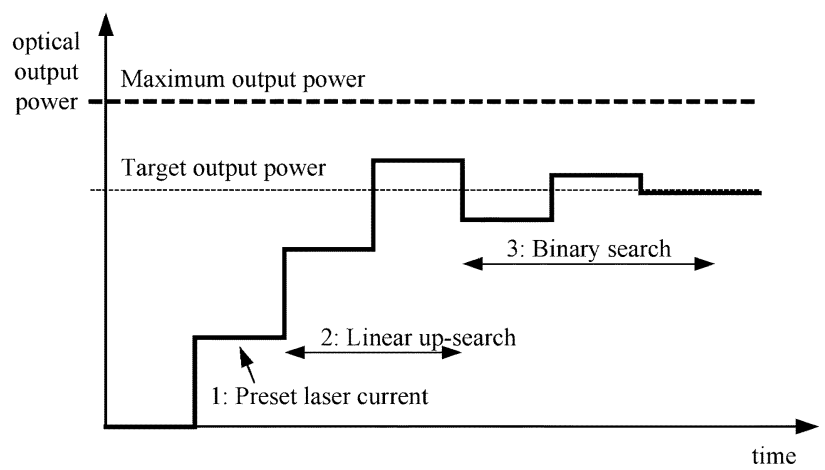

Fig. 2. Adapted binary search algorithm output example.

algorithm takes over the regulation. The algorithm typically needs only two upward steps and eight binary search steps. As a dual loop controls both the low and the high level, the total number of regulation steps is typically 20 . As soon as the binary search is finished, the APC reports a "Level OK" and automatically switches to a slow APC mode. In this mode, the algorithm adapts the laser driver currents in small steps to compensate for changes in the laser output power during operation. To speed up the initial power setup, the APC prepares two settings for every step in the algorithm, one for the case that the measured level would be too low and another for the case that the level would be too high. Once a new measurement is available, the correct setting can be applied almost immediately and a new measurement can start. The total time needed per algorithm step mainly consists of the settling time of the IBIAS and IMOD current sources and the level monitoring circuit delay. The digital calculation runs concurrently with these delays, which reduces the fast APC regulation time by a factor of more than two.

The APC adjusts the low and the high output level alternately. However, when the low-level IBIAS is adjusted, this also affects the optical high level. To be able to run the binary search algorithm for both levels simultaneously, an adjustment of IBIAS is subtracted from IMOD so that regulation of the low optical level has no effect on the high optical level. In the slow APC mode, a maximum one measurement per burst is performed and only small steps (e.g., $80 \mu \mathrm{A})$ are taken to not disturb the ongoing transmission. A message field with ten bytes in the PLOAMu is sufficient for performing a single measurement of both levels. Special care is taken to not fatally damage the laser diode by exceeding the maximum optical output. The BM-TX chip is equipped with three programmable registers to store an absolute maximum laser current at low, medium and high ambient temperature. An on-chip thermometer decides which of these values is selected, while the algorithm ensures that the total drive current never exceeds the maximum value.

\section{RESULTS}

The proposed APC scheme was realized in a $0.35-\mu \mathrm{m}$ bipolar CMOS burst-mode laser diode driver (BM-LDD) chip. The BM-LDD was tested with a Mitsubishi FU 445SDF laser diode. Fig. 3 shows a plot of the optical output power regulations and the "Level OK" signal during initialization, followed by a normal data burst with a PLOAMu overhead. Despite the very conservative preset levels, the APC can complete the fast 


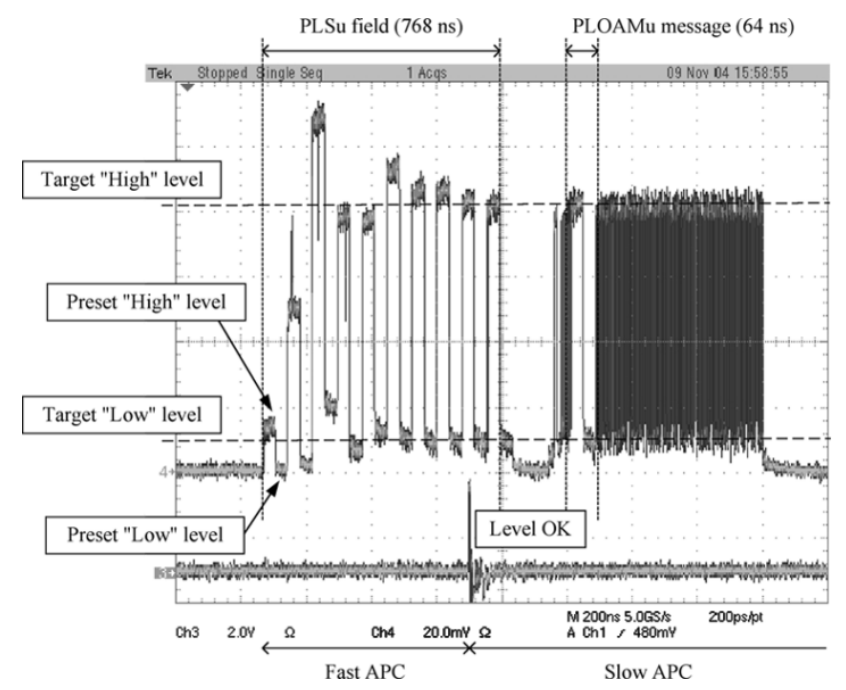

Fig. 3. APC algorithm: initialization burst with a PLSu field followed by a normal data burst with a PLOAMu message field.

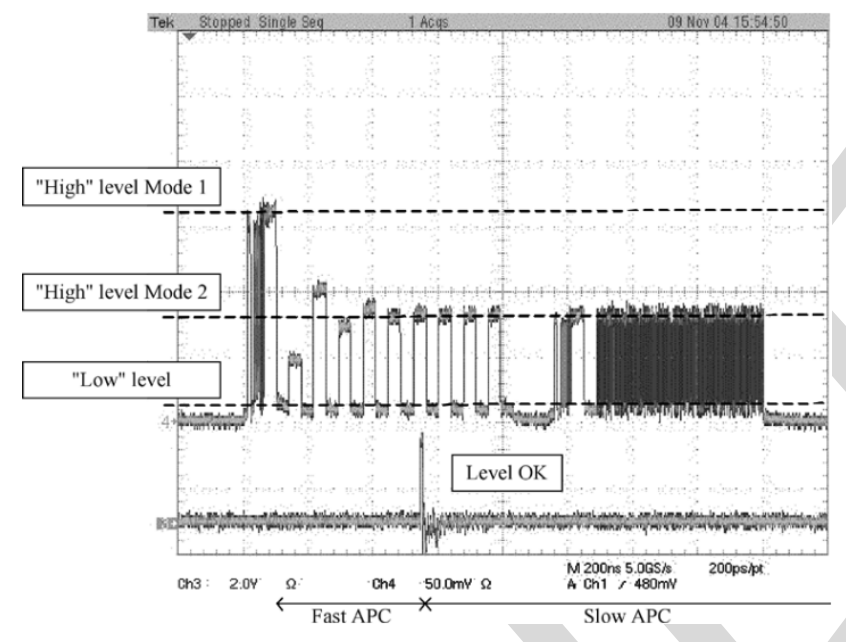

Fig. 4. BM-TX changes its mode from PLM Mode 1 to PLM Mode 2 in one PLSu field.

initialization within one PLSu field (768 ns). Fig. 4 displays the optical output power for an active BM-TX changing from PLM Mode 1 to PLM Mode 2. The optical "1" level is halved, whereas the " 0 " level is $-13.5 \mathrm{dBm}$ for all PLM states. The performance of the APC over temperature is shown in Fig. 5. Fig. 5(a) shows the average optical power, whereas Fig. 5(b) shows the ER. Varying the power supply in the $3.3 \mathrm{~V} \pm 5 \%$ range has only little influence $(0.13 \mathrm{~dB})$ and is not shown. The variation of the average output power over the temperature range is only $0.82 \mathrm{~dB}$, whereas $5 \mathrm{~dB}$ is allowed (Table I). This leaves a lot of margin for the use of cheaper optics. The variation of the ER in PLM Mode 2 is only $0.35 \mathrm{~dB}$, so that the ER is always well above $10 \mathrm{~dB}$. In PLM Mode 1 and PLM Mode 0 the ER is much higher as the " 0 " level is not increased. The variation of the ER is smaller than the variation of the average power, notwithstanding the fact that the " 0 " level is more difficult to control. This is because the tracking error of the PD has the same impact on both optical levels so that the ER is not influenced by the tracking error.

The worst-case power consumption of the complete APC loop is $116 \mathrm{~mW}$. In between nonconsecutive bursts, this power

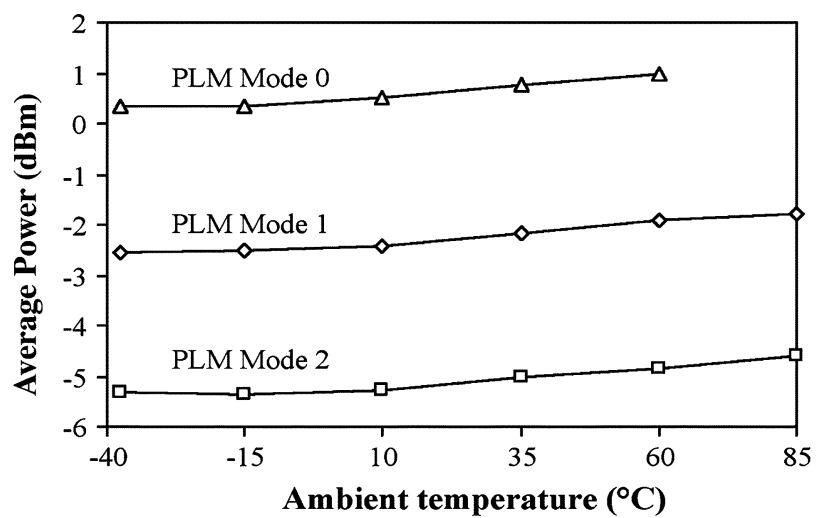

(a)

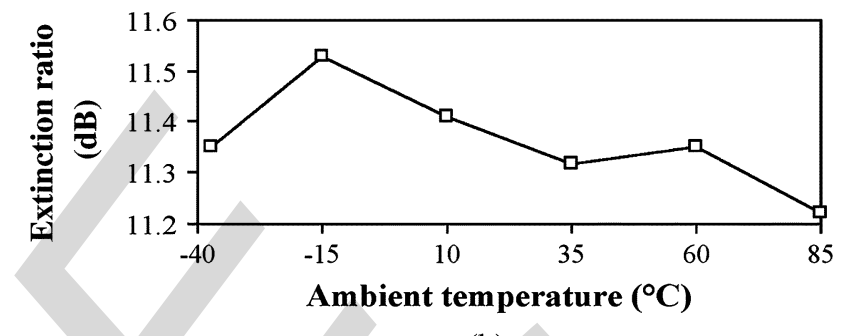

(b)

Fig. 5. (a) Measured output power at different PLM modes over temperature. (b) ER over temperature for PLM Mode 2.

consumption is only $29 \mathrm{~mW}$ by using the dedicated "Transmit Enable" signal.

\section{CONCLUSION}

An innovative APC concept for a GPON BM-TX has been proposed and implemented. The digital implementation of the APC algorithm makes the loop both accurate and fast. This concept is compliant to the GPON standard including the PLM option. Measurements show that the optical output of the BM-TX varies only $0.82 \mathrm{~dB}$ over the complete temperature range, whereas the ER varies only $0.35 \mathrm{~dB}$.

\section{REFERENCES}

[1] X. Z. Qiu, P. Ossieur, J. Bauwelinck, Y. C. Yi, D. Verhulst, J. Vandewege, B. De Vos, and P. Solina, "Development of GPON upstream physical media dependent prototypes," J. Lightw. Technol., vol. 22, no. 11, pp. 2498-2508, Nov. 2004.

[2] Gigabit-Capable Passive Optical Networks (GPON): Physical Media Dependent (PMD) Layer Specification, ITU-T Recommendation G.984.2, 2003.

[3] E. Säckinger, Y. Ota, T. J. Gabara, and W. C. Fischer, "A 15-mW, $155-\mathrm{Mb} / \mathrm{s} \mathrm{CMOS}$ burst-mode laser driver with automatic power control and end-of-life detection," J. Solid-States Circuits, vol. 35, no. 2, pp. 269-275, Feb. 2000.

[4] J. Bauwelinck, D. Verhulst, P. Ossieur, X.-Z. Qiu, J. Vandewege, and B. De Vos, "DC-coupled burst-mode transmitter for a $1.25 \mathrm{Gbit} / \mathrm{s}$ upstream PON," Electron. Lett., vol. 40, no. 8, pp. 501-502, Apr. 2004.

[5] Y. Park, C. Lim, and I. Jung, "ONU power equalization of ethernet PON systems," IEEE Photon. Technol. Lett., vol. 16, no. 8, pp. 1984-1986, Aug. 2004

[6] J. Bauwelinck, D. Verhulst, P. Ossieur, X. Z. Qiu, J. Vandewege, and B. De Vos, "Current mode circuits for fast and accurate optical level monitoring with wide dynamic range," IEICE Trans. Commun., vol. E87-B, no. 9, pp. 2641-2647, Sep. 2004.

[7] D. Verhulst, Y. Martens, J. Bauwelinck, X. Z. Qiu, and J. Vandewege, "Fast consecutive zero and one bits detection circuits for a 1.244 Gbps burst mode laser driver," IEICE Trans. Commun., vol. E87-B, no. 8, pp. 2377-2379, Aug. 2004. 